\title{
Verifique sus conocimientos sobre y la comunicación (TIC) en salud
}

Jordi Galimanya, Carles Rúbies ${ }^{\mathrm{b}}$ y Joan Guanyabens ${ }^{\mathrm{c}}$

a Enfermero. Profesor de la EUE de la Universidad de Barcelona, Barcelona, España

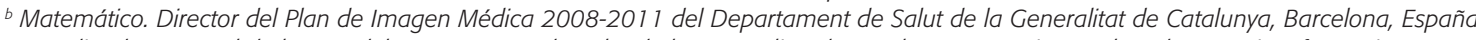

c Coordinador General de las TIC del Departament de Salut de la Generalitat de Catalunya. Consejero Delegado Agencia Información,

Evaluación y Calidad en Salud, Barcelona, España

\section{Introducción}

La presente entrega de la serie de Nursing sobre las pruebas complementarias está dedicada a las tecnologías de la información y la comunicación (TIC).

Las TIC plantean una nueva perspectiva en la integración de la información sanitaria en general y de las pruebas complementarias en concreto. Las historias clínicas electrónicas permiten disponer de los datos de salud de los pacientes en cualquier momento, lugar geográfico y nivel asistencial, y permiten que el profesional de enfermería acceda a esta información en tiempo real.

La digitalización de la información clínica del paciente en general, de las imágenes médicas y de los distintos registros, junto con la interoperabilidad y las redes de comunicación, son los elementos clave en este contexto caracterizado por la disminución de los recursos humanos y materiales, el incremento del gasto y la necesidad de conseguir eficiencia organizativa de los sistemas sanitarios.

Desde el punto de vista de los profesionales de enfermería las TIC plantean un doble reto. Por un lado, el reto formativo y su capacidad como herramienta de intercambio y compartición de la información y, por otro, el cambio que supone desde el punto de vista del paciente y su relación con el profesional de enfermería como referente del sistema sanitario.

Para conseguir esto son necesarios profundos cambios organizativos que permitan al profesional de enfermería adaptarse al nuevo entorno tecnológico para ofrecer unos cuidados de calidad a la comunidad.

1. Las tecnologías de la información y la comunicación (TIC) se definen como:

a. Guías para una buena comunicación entre el paciente y los profesionales de la salud.

b. El sistema de financiación que se está imponiendo en los sistemas sanitarios de los países desarrollados.

c. Herramientas tecnológicas diseñadas para facilitar la disponibilidad y el acceso a la información.

d. Se refieren a los departamentos de sistemas de información de los hospitales.
2. El objetivo final de la generalización del uso de las TIC en el campo de la salud es:

a. Investigar e innovar en las tecnologías centradas en patologías minoritarias.

b. Minimizar el impacto de las tecnologías sanitarias en los profesionales de la salud.

c. Implementar las tecnologías que mejoren la salud de las personas.

d. Minimizar el impacto de las TIC en los usuarios de los sistemas sanitarios.
3. El principal efecto derivado de la implementación de las TIC en los sistemas sanitarios para los pacientes es:

a. La reducción de la calidad asistencial debido al incremento de los costes provocados por la incorporación de las TIC.

b. Ninguna; únicamente tiene consecuencias sobre los profesionales de la salud.

c. La mejora asistencial, porque los ordenadores son más eficientes que las personas.

d. La mejora asistencial como consecuencia de disponer o tener acceso a más y mejor información.

4. El principal impacto que tiene la implementación de las TIC en los cuidados de salud para el profesional de enfermería es:

a. El aumento de las listas de espera, el aumento de la demanda y de la presión asistencial.

b. El cambio en los procesos asistenciales debido a los cambios en la obtención y el registro de la información sanitaria del paciente.

c. El cambio en la relación que se establece entre el profesional sanitario y el usuario del servicio de salud.

d. La disminución de las cargas de trabajo para los profesionales sanitarios.

5. ¿A qué hace referencia el concepto de integración de la información y 


\section{0 \\ edición española}

\section{las tecnologías de la información}

los datos de salud del paciente que se registran y almacenan?

a. A la existencia de diversos y variados sistemas independientes, sin relación entre sí, de registro y almacenaje de la información del paciente.

b. A la capacidad de integrar en una sola plataforma o dispositivo tecnológico toda la información sanitaria del paciente.

c. A la necesidad de reducir la información y los registros sanitarios de los pacientes.

d. Al aumento de la información sanitaria y los datos de salud de los pacientes que supone la incorporación de las TIC en los sistemas sanitarios.

6. El desarrollo de sistemas de innovación basados en las TIC

y la E-Salud supone:

a. Incrementar la equidad, mejorar la continuidad asistencial y fomentar el autocuidado en los pacientes.

b. Disminuir la continuidad asistencial y limitar el autocuidado en los pacientes.

c. Reducir los recursos dedicados a salud para dedicarlos a innovación.

d. Reducir la eficacia, la eficiencia y la sostenibilidad de los sistemas sanitarios.

7. La historia clínica electrónica (HCE) de salud se define como:

a. Un sistema informatizado de prestación de cuidados al paciente utilizado en los países desarrollados. b. La versión digital de la historia clínica tradicional en papel.

c. Un sistema de registro avanzado de los cuidados que el profesional de enfermería realiza al paciente.

d. Un sistema de prescripción electrónica de medicación.

8. Las principales ventajas de la historia clínica electrónica (HCE) en comparación con la historia clínica tradicional en papel son:

a. Que la información está estructurada para facilitar el acceso a la misma.

b. La reducción de tiempos para encontrar la información necesaria para una correcta asistencia.

c. La disponibilidad de mayor cantidad de información.

d. Todas las respuestas anteriores son correctas.

9. ¿A qué se refiere el término "historia clínica compartida"?

a. Un sistema de información que permite al profesional de salud enviar datos de sus pacientes a foros de debate.

b. Un sistema de información que permite, a todo el que se registre previamente, acceder a la información clínica de dicho sistema.

c. Un sistema de información que aglutina las historias clínicas de varios centros.

d. Un sistema similar al Facebook, donde se puede encontrar información clínica del paciente que se está tratando.
10. ¿A qué hace referencia el término "historia clínica personal"?

a. A la historia clínica del personal del centro de salud.

b. A la historia clínica de cada uno de los ciudadanos.

c. A la historia clínica de cada uno de los pacientes.

d. A la parte de la historia clínica que no queremos que sea pública.

11. La digitalización de la imagen médica supone para los sistemas de salud:

a. Mayor accesibilidad y disponibilidad a las exploraciones de los pacientes.

b. Reducir las exploraciones porque se evitan repeticiones de las mismas.

c. Abaratar costes en general, en todo el proceso de diagnóstico.

d. Todas las respuestas anteriores son correctas.

12. Las principales ventajas de la imagen en formato digital en comparación con el formato analógico son:

a. Proporciona mayor continuidad asistencial al poder visualizar la imagen en varios sitios a la vez sin pérdida de tiempo.

b. La reducción del número de repeticiones de exploraciones, ya que la imagen se puede retocar y puede circular entre los distintos centros de salud.

c. La posibilidad de post-proceso de la imagen en las estaciones de trabajo de los profesionales. 
d. Todas las respuestas anteriores son correctas.

13. Las consecuencias que tiene la digitalización de la imagen médica para los profesionales de enfermería son que:

a. Facilita el desarrollo de plataformas de conocimiento basadas en la compartición de la información.

b. Supone la reducción drástica de formación para los profesionales de enfermería.

c. El uso y gestión de los estudios digitalizados es muy complejo.

d. Supone una importante barrera para trabajar de manera colaborativa.

14. Respecto a la prescripción electrónica de medicamentos, es cierto que:

a. Es un mecanismo de optimización que permite reducir a la mitad el gasto sanitario dedicado a farmacia.

b. Es un instrumento que permite tener información de todo el proceso de prescripción y su seguimiento.

c. Es una herramienta que dispara el consumo de medicamentos.

d. Representa importantes riesgos para la seguridad del paciente.

15. ¿Cuál cree que será la influencia que tendrá la implementación de las TIC en los sistemas sanitarios para la salud de las personas?

a. La atención sanitaria conseguirá mayor acercamiento a la población, permitiendo un mejor acceso y más equidad para los ciudadanos.

b. Los pacientes verán reducidos los contactos con su profesional de enfermería de referencia.

c. Los servicios de salud se redefinirán teniendo en cuenta las necesidades tecnológicas de los sistemas sanitarios.

d. Los cuidados de salud se caracterizarán por ser todos virtuales, gracias a diferentes plataformas tecnológicas.

\section{Respuestas}

1 c. Las tecnologías de la información y la comunicación (TIC) son el conjunto de tecnologías que proporcionan la información, las herramientas para su proceso y los canales de comunicación. Dichas tecnologías permiten la adquisición, producción, almacenamiento, tratamiento, comunicación, registro y presentación de dicha información

En el caso de su aplicación en el ámbito sanitario, tienen como objetivo primordial favorecer más y mejor el acceso al sistema sanitario y sus diferentes recursos, con la información clínica relevante del usuario como máximo exponente. Además propician un entorno en el que se comparte la información por parte de todos los agentes implicados en el proceso de salud del ciudadano, hecho que es clave para el profesional de enfermería en el desarrollo de su práctica asistencial en cualquier ámbito en el que se produzca. Además, tiene relevancia en otros ámbitos como la docencia, la gestión o la investigación, en los que el profesional de enfermería participa de manera activa.

2 c. El principal objetivo de un sistema sanitario es proporcionar cuidados de salud a los ciudadanos, manteniendo y promocionando los niveles de bienestar de la población.

En este sentido las TIC, como dispositivos capaces de facilitar y mejorar muchos de los procesos asistenciales, pueden contribuir a dicho objetivo. Para que las TIC sean efectivas han de plantearse y entenderse desde la perspectiva de su efectividad para facilitar y ayudar a mejorar procesos optimizando protocolos y siempre teniendo en cuenta que el foco de atención es el paciente.

El objetivo de proporcionar salud a la población está muy condicionado por factores y elementos coyunturales que hacen imprescindible que los sistemas sanitarios puedan responder a las expectativas relacionadas con la salud de manera eficaz. En este papel, las TIC juegan un papel primordial si se aprovecha su potencial.

3 d. La principal consecuencia de la implantación de las TIC en los sistemas sanitarios es la mejora asistencial en general. Ésta es consecuencia de un mayor acceso a la información relacionada con la salud del paciente. Esto supone, para los usuarios de los sistemas sanitarios, la posibilidad de recibir una atención más personalizada.

Algunos ejemplos concretos de la trascendencia que están teniendo las TIC en la salud de las personas son, por ejemplo, la posibilidad de diagnosticar alteraciones de la salud precozmente, permitiendo adoptar medidas terapéuticas inmediatas. Los datos demuestran que se ha reducido la mortalidad por causa de infarto cuando se dispone de sistemas de electrocardiografía avanzados para detectar alteraciones cardíacas (Código Infarto). También, en el caso del Código ICTUS, la posibilidad de un diagnóstico rápido permite medidas terapéuticas inmediatas que minimizan y reducen las afectaciones.

Por parte del paciente se requiere una actitud más proactiva, en contraposición con la que tradicionalmente se ha caracterizado por la pasividad o, como mucho, la reactividad, fomentada en algunos casos por el propio sistema sanitario y sus profesionales.

Por tanto, se puede afirmar que, en general, las ventajas para el usuario son numerosas, y esto fomenta una percepción de calidad en el proceso asistencial. Sin embargo, el profesional de enfermería debe tener en cuenta el cambio que todo ello supone desde el punto de vista de la relación entre el usuario y el sistema sanitario. 
4 b. El nuevo contexto asistencial con el uso de las TIC supone para el profesional de enfermería, más allá de disponer de la información del paciente, poder acceder a sistemas de compartición del conocimiento. Esto facilita la interconsulta o el asesoramiento entre los profesionales de enfermería, con más interrelación y posibilidad de estudiar y usar sistemas de cuidados basados en la teleenfermería. Por tanto, se puede trabajar en un entorno de trabajo colaborativo, caracterizado por la comunicación e interconexión permanente entre los agentes implicados en el proceso de salud del paciente. Éste es un aspecto bastante novedoso que rompe con la concepción de información compartimentada, propiedad de un centro o nivel asistencial. Esto supone, además, un importante valor añadido a la labor del profesional de enfermería que ha de repercutir, de manera positiva, en la calidad de la asistencia prestada a la comunidad.

Esto no debería suponer un cambio en la relación que se establece entre el profesional sanitario y el usuario del servicio de salud; se debe evitar la despersonalización de la atención, intentando que los pacientes estén más informados e implicados en su proceso asistencial.

5 b. La integración de toda la información de salud relacionada con un paciente es una de las claves y uno de los principales objetivos que deben perseguir las herramientas TIC aplicadas a la sanidad.

Para que el profesional de enfermería pueda proporcionar unos cuidados de salud integrales, de calidad y con la máxima seguridad y eficacia para el paciente, es necesario disponer de toda la información sanitaria en un único acceso seguro, fiable y rápido, independientemente de la entidad proveedora de servicios de salud que haya generado esa información. Información integrada significa, por ejemplo, que un paciente que acude al centro de atención primaria de urgencia puede tener toda su información sanitaria exactamente igual de accesible que un paciente que tiene una visita programada hace varios días.

Por tanto, la integración de la información relacionada con la salud y la enfermedad del paciente es otro de los grandes retos que plantea la asistencia de la salud moderna: disponer de forma fácil y a la vez segura de toda la información relevante del paciente, en el momento y sitio requeridos, independientemente de la fuente de información.

6 a. Las innovaciones en el campo de las TIC relacionadas con la salud deben tener muy en cuenta el actual contexto sociosanitario, caracterizado por la escasez de recursos, la necesidad de mantener la equidad, el objetivo de mejorar la continuidad asistencial y fomentar el autocuidado en los pacientes. Los dispositivos y mecanismos TIC deben tener un claro impacto en la mejora de la salud de las personas, y para ello se deben focalizar en conseguir la mejora de los procesos asistenciales.

La introducción de las TIC en el ámbito sanitario no es un proceso aislado, sino que se enmarca en el contexto general de innovación y desarrollo que, en las últimas décadas, está afectando a muchos campos del conocimiento y de los servicios. En el sector salud es un fenómeno relativamente reciente pero muestra una rápida velocidad de progresión. Fruto de esto, las TIC se han convertido en un importante sector empresarial que, en el contexto económico actual, puede actuar como un sector generador de valor.

Por tanto, invertir en sistemas innovadores que tengan como punto de partida o base las TIC mejora las relaciones entre todos los agentes de salud, favorece la continuidad asistencial, fomenta el cuidado de la propia salud y sirve de apoyo a las actividades de los profesionales sanitarios.

7 b. La HCE es la versión digital o electrónica de la historia clínica en papel. La historia clínica es el instrumento que los sistemas de prestación de cuidados de salud utilizan para registrar la relación establecida entre el usuario y el sistema. En este caso, la HCE registra de manera electrónica la información relevante y permite el acceso a diferentes registros, exploraciones, etc. Es, por tanto, el repositorio donde se registra la información referente a la salud y la enfermedad del paciente. En general, se registran los aspectos más relevantes referidos a la salud del paciente y, teniendo en cuenta que accederán a ésta diferentes personas en diferentes centros asistenciales, se requiere cierta uniformidad y consenso en lo que se registra y en cómo se registra en la HCE.

Hay que tener en cuenta que la información y los datos de los pacientes que se registran en la HCE no son exactamente los mismos que los registrados en la historia clínica en papel. Igualmente, dependiendo del diseño de la HCE, pueden variar sus contenidos aunque existan unos mínimos básicos que son comunes a la mayoría de ellas. Esto hace que sean bastante coincidentes en cuando a la información a la que dan acceso al profesional de enfermería.

8 d. La informatización aporta a los datos contenidos en la HCE, además de una uniformidad y una estructuración, la posibilidad de almacenar mayor cantidad de datos. Teniendo en cuenta el espacio físico que ocupan los historiales de salud en papel en los centros sanitarios, es fácil deducir el coste que esto supone. Desde un punto de vista de seguridad es importante considerar que las HCE ofrecen la posibilidad 
de registro y seguimiento de las consultas realizadas por cualquier profesional.

Es por todo esto que el manejo de datos de salud informatizados que posibilita la HCE tiene además un impacto positivo en los profesionales de enfermería que utilizan estos dispositivos teniendo en cuenta que favorece la formación, la investigación y la evolución de los cuidados de enfermería, puesto que se pueden desarrollar plataformas de conocimiento de las que se pueden beneficiar los profesionales de enfermería y, en este sentido, todavía está por explorar el potencial que esta nueva posibilidad permite a los sistemas sanitarios y los profesionales que forman parte de ellos.

Por tanto, la gran ventaja de la HCE es la posibilidad y la rapidez de acceder a los datos y la información desde cualquier punto geográfico, en cualquier momento o desde cualquier nivel asistencial. Sin duda, esto supone para el profesional de enfermería una importante herramienta para conocer con concreción los datos, registros y otros elementos o informaciones destacadas del paciente al que debe prestar atención o cuidados.

9 c. El término HCE hace referencia a la historia clínica electrónica del paciente en un ámbito concreto. En general, se enmarca en un centro o grupo de centros gestionados por una misma entidad proveedora de servicios de salud.

El término historia clínica compartida (HCC) hace referencia a la compartición de la historia clínica entre distintas entidades proveedoras. En general, tendremos una HCE en cada centro y una HCC por encima de los centros con la información de salud más relevante para cada paciente.

La interoperabilidad es una de las claves de cualquier sistema de compartición de la información. Este concepto hace referencia a la capacidad de diversos dispositivos para entenderse entre ellos, es decir, a la capacidad de intercambiar información. Para ello es preciso que estén diseñados o pensados teniendo en cuenta este objetivo, con base a protocolos y lenguajes informáticos estandarizados para facilitar posteriormente la comunicación sin necesidad de nuevos dispositivos que interpreten y comuniquen la información entre un dispositivo y otro. Hay que tener en cuenta que muchos de estos dispositivos se utilizan más allá de las fronteras o jurisdicciones administrativas $y$, por tanto, es básico que puedan intercomunicarse entre ellos.

10 b. Anteriormente se ha definido la HCE y la HCC. Existe otro concepto, que es la historia clínica personal (HCP), definida como la historia clínica de cada uno de los ciudadanos, gestionada por él mismo. La HCE está gestionada por el centro asistencial, la HCC está gestionada por el ente que facilita la compartición entre centros y la HCP está gestionada por el propio ciudadano, incorporando los elementos que considere importantes a su historia clínica. Se puede entender como un cajón donde se guardan los informes, las placas, los estudios, las anotaciones, etc., para gestionar adecuadamente nuestra salud. La HCP está todavía en sus incipientes orígenes. Una vez se consoliden las HCE y las HCC, vendrá el auge de la HCP.

Uno de los grandes problemas de los sistemas sanitarios es el acceso equitativo por parte de los ciudadanos, sin listas de espera excesivas e intentando que el usuario tenga una percepción de calidad en todo el proceso. Con el panorama socioeconómico actual, parece que este objetivo únicamente será asumible partiendo de una concepción más participativa y con más responsabilidad de los usuarios. Ésta es una de las oportunidades que ofrecen las TIC y la E-Salud, junto con la mejora de la gestión de recursos para que sea más eficaz y se supere la ineficiencia actual.

11 d. La digitalización de la imagen médica supone, sobre todo, la posibilidad de mejorar el acceso a las exploraciones médicas basadas en la imagen. Los sistemas digitales de producción, almacenaje y distribución de la imagen médica permiten mayor disponibilidad de los estudios. Dicha disponibilidad supone, a su vez, mayor facilidad de interconsulta, visionado remoto de imágenes y estudios, etc. Desde un punto de vista del profesional de enfermería, y pensando en la actividad asistencial, hay que tener en cuenta el importante cambio de mentalidad que supone este entorno de trabajo con la imagen médica digitalizada. Se requerirán algunos cambios, en este sentido, para aprovechar las posibilidades que brindan la telerradiología, el telediagnóstico o la telemonitorización para mejorar los cuidados a los pacientes, que son ya en la actualidad muy relevantes, con numerosos ejemplos prácticos de los mismos.

12 d. Tal como se ha descrito en la pregunta 11, el uso de imágenes digitalizadas en los centros de salud ha revolucionado la manera de entender la asistencia y la prestación de los cuidados. Las ventajas de este nuevo entorno asistencial para el paciente son la reducción de repeticiones, esperas y desplazamientos innecesarios, aportando mayor continuidad asistencial a todo su proceso asistencial, gracias a la posibilidad de visualizar la imagen en varios sitios a la vez sin pérdida de tiempo. Por ejemplo, en pacientes que se trasladan de un centro sanitario a otro, la información generada en el centro sanitario de origen se puede visionar y utilizar sin necesidad de repeticiones en el centro de destino.

Existen pocas desventajas del proceso de digitalización de la 
imagen médica; pese a ello, es cierto que en los departamentos de nueva creación o en los que se renuevan o se pasa de sistemas analógicos a sistemas digitales, hay que tener en cuenta la inversión inicial que suponen los dispositivos necesarios para ello. Sin embargo, las experiencias y los estudios publicados acerca del tema demuestran que ésta se ve claramente compensada.

13 a. En la misma línea que ocurre con los datos de salud del paciente, en el caso de la imagen se puede extrapolar también la posibilidad de desarrollar plataformas de conocimiento basadas en la compartición de la información.

Uno de los aspectos destacados en este contexto es la necesidad de formación. Cuando se incorporan nuevas herramientas para desarrollar las tareas profesionales se han de valorar seriamente las implicaciones que supone en cuanto a la formación de los profesionales que las utilizarán. Se pueden definir personas de referencia en los equipos de enfermería, que actúan como referente ante problemas, dudas o actividades formativas. También en este sentido, se puede aprovechar el desarrollo tecnológico de los dispositivos para favorecer la evolución de los cuidados de enfermería teniendo en cuenta la posibilidad de compartir la información, en este caso de las imágenes médicas pero en general con los datos del paciente.

El profesional de enfermería debe ser, desde el desarrollo hasta la implementación y posterior seguimiento o mantenimiento, una pieza clave desde el punto de vista no únicamente de su utilización como usuario, sino también desde el punto de vista del liderazgo del proceso.

14 b. La prescripción electrónica, la dispensación electrónica de medicación o receta electrónica es un instrumento que posibilita una gestión de todo el proceso, de por sí complejo y con necesidad de elevada fiabilidad y seguridad, con garantías para el usuario. El sistema ofrece más seguridad y control y, por tanto, el paciente se puede responsabilizar y participar para tomar conciencia de la gestión de su medicación desde un punto de vista más integral. Además de las ventajas para los usuarios, las ventajas para las administraciones y para los profesionales que el sistema de dispensación electrónica ofrece son también evidentes. Por tanto, se puede afirmar que la prescripción electrónica es un instrumento que, en general, permite disponer de información del proceso de prescripción y hacer un seguimiento del mismo.

15 a. Las TIC son dispositivos capaces de hacer más fácil, más efectivo y más sostenible el sistema sanitario, con el fin de mantener y mejorar los niveles de salud de la comunidad. Por tanto las TIC son, en sí mismas, dispositivos muy potentes de gestión de la información capaces de facilitar y mejorar muchos de los procesos asistenciales que actualmente se desarrollan en los sistemas sanitarios. Sin embargo, las TIC no son la solución si no se entienden desde la perspectiva de su efectividad para facilitar y ayudar a mejorar procesos, optimizar protocolos, con el foco en el usuario como máximo protagonista de la relación asistencial.

La E-Salud es un campo emergente en el mundo de la salud igual que lo ha sido en otros campos de la sociedad en los que la comunidad tiene participación. Se está consolidando como una de las vías más efectivas para conseguir que los sistemas sanitarios sean más equitativos. Los principales frentes en los que se están invirtiendo esfuerzos son la manera de mejorar la continuidad asistencial, como elemento clave para mejorar el acceso, y cómo se puede conseguir que los usuarios se responsabilicen y tomen protagonismo en su salud.
Éstas son maneras de conseguir hacer más universal la prestación de salud a la comunidad sin que suponga un coste inasumible para los sistemas sanitarios.

En definitiva, los cuidados de salud a los pacientes se caracterizarán por un mayor acercamiento a la población, permitiendo un mejor acceso y más equidad para los ciudadanos.

\section{Bibliografía}

Cerdà I, Continente M, García C, Guanyabens J. Carpeta personal de salud. Med Clin (Barc). 2010;134 Supl 1:63-6.

Cugota L. TicSalut, la revolució digital al servei de les persones. Exemples d'innovació al Sistema Català de Salut. Barcelona: Infonomia; 2007.

Donoso L, Martínez C. Sistemas de información en radiología. En: Radiología Esencial. 1. ${ }^{a}$ ed. Madrid: Panamericana; 2010. p. 1825-38.

Galimany J, Girbau MR, Salas K. Telecuidado, una visión asistencial. Rol. 2010;33(11):17-20.

Galimany J, Guanyabens J. Las tecnologías de la información y la comunicación en salud. En: Atención familiar y salud comunitaria. Conceptos y materiales para docentes. $1 .^{\mathrm{a}} \mathrm{ed}$. Barcelona: Elsevier; 2011. p. 251-60.

Girbau MR, Galimany J, Salas K. Cuidados de enfermería y las tecnologías de la información y la comunicación. Nursing. 2010;28(1):56-9.

Hayrinen K, Saranto K, Nykanen P. Definition, structure, content, use, and impacts of electronic health records: A review of the research literature. Int J Med Inform. 2008;77:291-304.

Jha AK, Doolan D, Grandt D, Scott T, Bates DW. The use of health information technology in seven nations. Int J Med Inform. 2008;77:848-54.

Marimon S, Rovira M, Acedo M, Nozal MA, Guanyabens J. Historia clínica compartida en Cataluña. Med Clin. 2010;134 Suppl 1:45-8

Poissant L, Pereira J, Tamblyn R, Kawasumi Y. The impact of electronic health records on time efficiency of physicians and nurses: A systematic review. J Am Med Inform Assoc. 2005;12(5):505-16

Rúbies C, Salas T, Moya F, Guanyabens J Imagen médica, telemedicina y teleasistencia médica. Med Clin (Barc). 2010;134 Supl 1: 56-62.

Correspondencia: Jordi Galimany Masclans Departament d'Infermeria de Salut Pública, Salut Mental i Materno-Infantil

Campus de Bellvitge. Pavelló de Govern. 3. a planta C/ Feixa Llarga s/n. 08907 L'Hospitalet de Llobregat. Barcelona. España

Correo electrónico: jordigalimany@ub.edu 